\title{
A brief cross-cultural ethnobotanical note on the Abotani tribes of Arunachal Pradesh, India
}

\author{
Hage Yanka ${ }^{1}$, Rubu Rinyo ${ }^{1}$, Sanjib K. Das ${ }^{2}$, Tridip J. Das², Dipayan Paul ${ }^{2}$, \\ Debmalya Das Gupta², Pallabi K. Hui ${ }^{2}$, Sapana Bansod ${ }^{3}$, Chandraiah Godugu ${ }^{3}$, R. \\ Ananthan ${ }^{5}$, Sanjay Jambhulkar ${ }^{4}$ and Hui Tag ${ }^{1,6}$ \\ 'Plant Systematic and Ethnobotanical Research Laboratory, Department of Botany, Rajiv Gandhi \\ University, Rono Hills, Doimukh-791112, Arunachal Pradesh, India. \\ ${ }^{2}$ Department of Biotechnology, National Institute of Technology - Arunachal Pradesh, Yupia-791112, \\ District Papum Pare, Arunachal Pradesh, India \\ ${ }^{3}$ Department of Regulatory Toxicology, National Institute of Pharmaceutical Education \& Research, \\ Balanagar, Hyderabad-500037, Telangana State, India \\ ${ }^{4}$ Nuclear Agriculture \& Biotechnology Division, Bhabha Atomic Research Centre, Trombay, Mumbai- \\ 400085, India \\ ${ }^{5}$ National Institute of Nutrition, Jamai-Osmania PO, Hyderabad-500007, Telangana State, India \\ ${ }^{6}$ Corresponding author; e-mail: huitag2008rgu@gmail.com
}

[Received 12.10.2019; Revised 21.12.2019; Accepted 22.12.2019; Published 31.12.2019]

\begin{abstract}
This paper discusses the brief cross cultural ethnobotany of the Abotani tribes of Arunachal Pradesh which comprises five different indigenous tribal communities, namely Adi, Apatani, Nyishi, Galo and Tagin residing in Arunachal Pradesh, India. A brief ethnobotanical survey was conducted in Abotani Biocultural Landscape (ABL) in 11 districts of Arunachal Pradesh during the year $2018-2019$ with fundamental aims to document significant ethnobotanical plants commonly used among all the 05 Abotani tribes in their local biocultural landscape and to document traditional knowledge and skills related to utilization of ethnobotanical plants resources using semi-structured questionnaire format. The survey have revealed impressive number of 45 species of ethnobotanically significant species frequently harvested and used as local food and medicine security as well as cultural materials among the local residents of 05 major tribes of Abotani cultural group residing in Siang, Subansiri, Kurung Kumey, Pakke Kessang and Papum Pare belt of Arunachal Pradesh.
\end{abstract}

Key words: Abotani Tribes, Arunachal Pradesh, Ethnobotanical notes, Conservation, Utilization

\section{INTRODUCTION}

The state of Arunachal Pradesh lies in the Northeastern region of India which is known to be a paradise for ethnobotanist and anthropologist (Mao \& Hyniewta 2000). The state is a part of as Himalayan Hotspot recognized by IUCN endowed with rich biodiversity and amazing variety of local culture and dialects nurtured and spoken by 26 major tribes and 110 subtribes in their traditional biocultural landscape (Myers et al. 2000; Mittermeir et al. 2005; Tag 2007). The Abotani tribes of Arunachal Pradesh comprises of Adi, Apatani, Nyishi, Tagin and Galo is an interesting group of indigenous tribal communities belonging to the mongoloid racial stock and speaks Tibeto-Burman dialect group devoid of written script but maintain their folk literature and culture through oral traditions since time immemorial (Nabam 2013; Tag 2014, 2017). The Abotani tribes primarily resides in 14 districts of Arunachal Pradesh, namely, Kamle, Kra Daadi, Kurung Kumey, East Kameng, Pakke Kesang, Papum Pare, 
East Siang, West Siang, Upper Siang, Lower Siang, Lower Dibang Valley, Lepa Rada, Lower Subansiri, and Upper Subansiri districts which falls within Eastern Himalaya region of India with an estimated population of over 450,000 souls (Census 2011). Abotani group of tribes considered mythical father Abotani as their primeval ancestor who pioneered Abotani languages, folk culture and oral literature tradition, traditional jhum agriculture, animal husbandry, fishing and hunting during ancient days (Showren 2009; Nabam 2013; Tag et al. 2008; Tag, 2018). Although Abotani groups of tribes and their traditional biocultural landscape are considered to be rich in biodiversity and ethnobotanical knowledge heritage, but only few publications are available to date which includes ethnobotanical appraisal on Adi of East Siang by Das (1986), Tag et al. (2008), Boko \& Narsimhan (2014), Momang et al. (2018a,b); ethnobotany of the Nyishi of Lower Subansiri and Kamle by Pal (1993), Tag et al. (2005), Tag \& Das (2007), Murtem (2000), Murtem \& Chaudhry (2016); ethnobotany of the Galo of West Siang by Omem et al. 2016), Gode et al. 2018; ethnobotany of the Tagin by Rinyo et al. (2018); ethnomedicinal reports on the Apatani tribe of Lower Subansiri by Bipul et al. (2017) whereas the nutritional aspects of some selected edible plants used by the Abotani tribes were reported by Pallabi et al. (2014). However, no reliable literatures are available to date on comparative ethnobotanical discourses on Abotani tribes based on cross cultural approaches. Therefore, present paper discusses ethnobotanical heritage of the five tribes of Abotani group of tribes based on brief field survey report presented on each tribes focused on few significant food, medicinal and cultural plants in cross cultural approach which has the immense economic and cultural values to the tribes under investigation for future conservation priorities and sustainable utilization.

\section{MATERIALS AND METHODS}

\section{Study area and ethnic culture}

The target study - State of Arunachal Pradesh is located between $26^{\circ} 28^{\prime} \mathrm{N}-29^{\circ} 30^{\prime} \mathrm{N}$ latitude and $91^{\circ} 30^{\prime} \mathrm{E}-97^{\circ} 30^{\prime} \mathrm{E}$ longitude covering total geographical area of 83,743 sq km. In the present study, only 11 districts namely, Kamle, Kra Daadi, Pakke Kesang, Papum Pare, East Siang, West Siang, Upper Siang, Lower Siang, Lower Dibang Valley, Lower Subansiri, and Upper Subansiri Districts which are primarily inhabited by the Abotani groups of tribes were selected for a rapid ethnobotanical survey. Name of the villages targeted and visited for ethnobotanical data collection were Dukum, Raga, Yada (Kamle district), Pakkekessang HQ, Langpong (Pakke Kesang district), Doimukh, Sagalee, Hoj, Naharlagun, Itanagar, Rono (Papum Pare district), Pasighat, Ayeng, Niglok, Mirbuk (East Siang district), Aalo, Tadin, Kombo (West Siang district), Geku, Perem, Bum, kumku (Upper Siang district), Roing, Jia, Bolung, Parbuk (Lower Dibang Valley district), Yazali, Peni, Tepin Hapa, Posa, Joram, Ziro, Hapoli, Siiro, Hari, Bula, Tajang, Dutta, Hija, Mudang Tage, Bamin Michi, Hong (Lower Subansiri district) Daporijo, Dumporijo, Uli, Bullo, Nguki (Upper Subansiri district). The forest of these 11 districts are rich in plant diversity which receive heavy rainfall during Northeast and Southwest Monsoon with annual relative humidity ranges between $80 \%-85 \%$. The tropical rain forest ranging up to $1000 \mathrm{~m}$ where under growth are quite thick and dense, and subtropical $(1000-2000 \mathrm{~m})$, temperate $(2000-$ $3000 \mathrm{~m})$ and alpine forest $(3000-4000 \mathrm{~m})$ has their unique vegetation characteristics which support different life-forms or habit groups (Kaul \& Haridasan 1987).

The major tribal communities found in the Abotani biocultural landscape are the followers of Donyi-Poloism sect of indigenous religion professed by Shaman called $N y u b /$ Nyubu or Miirii predominant in Arunachal Pradesh. The Nyishi is the largest among the Abotani groups of tribes residing in 7 districts of central Arunachal Pradesh who celebrates 


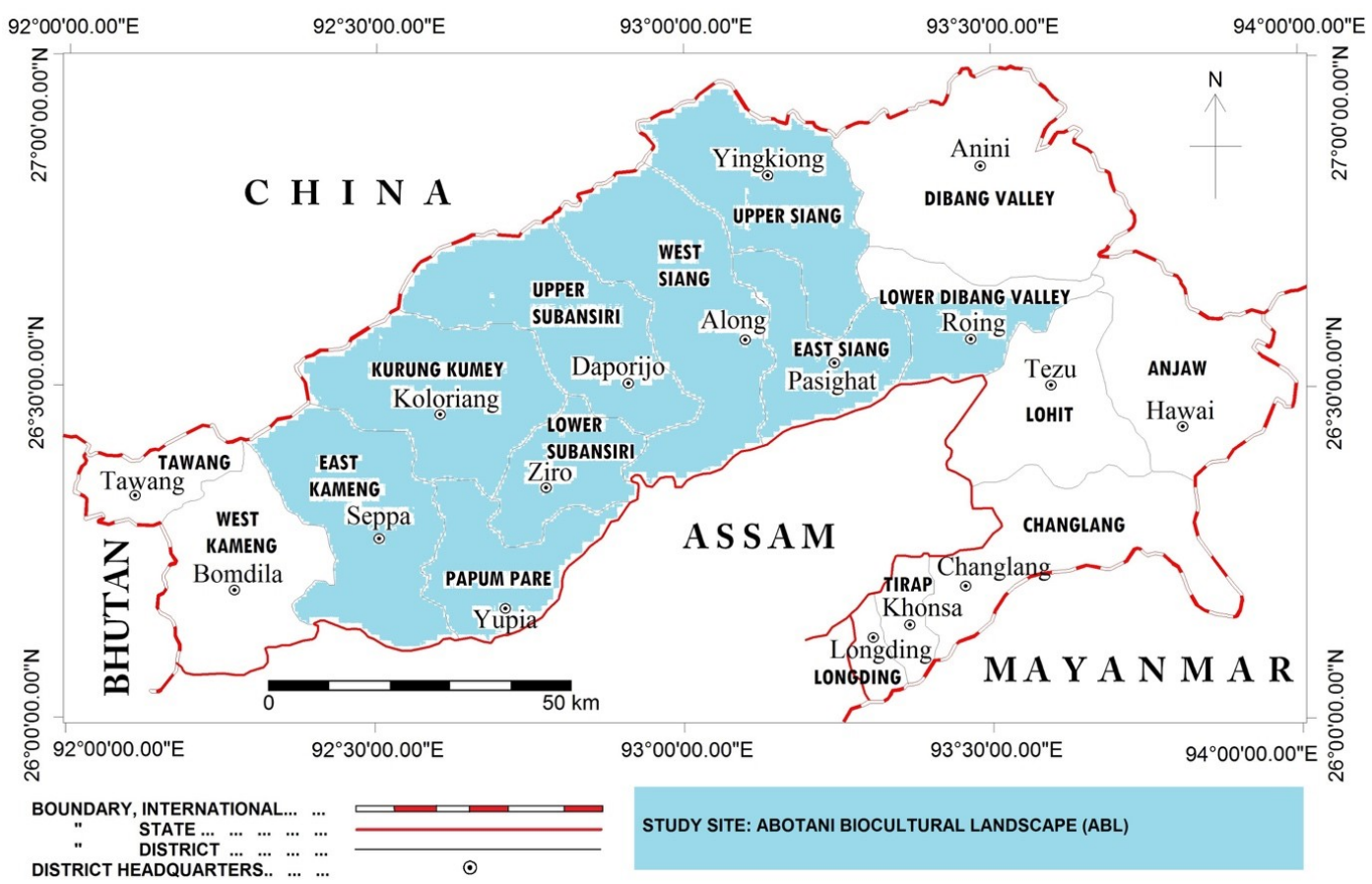

Figure 1. Map of Arunachal Pradesh showing study site - Abotani Biocultural Landscape (ABL) where cross cultural ethnobotanical survey was conducted

Boori Boot Yullo, Nyokum Yullo and Longte Yullo. The Apatani of Lower Subansiri celebrates Myoko and Murung in winter and Dree in summer month, whereas the Adi of Siang belt celebrates Aran and Solung, the Galo of West Siang, Lower Siang and Lepa Rada belt celebrates Mopin and Podii Barbii, and the Tagin of Upper Subansiri celebrates Shii Donyi festival. The celebration of the local festivals of all the Abotani groups of tribes begins in the month of January to February as they believe that during these winter months, the Gods and Goddesses have the warmest disposition towards the earth dwellers. The phase wise celebration of these local festivals culminates in the month of September is celebrated with fundamental aims to welcome new harvesting season and to dedicate their ritual prayers to the entire forces of the cosmos, almighty Donyi-Polo Gods, benevolent and malevolent demi-gods and goddesses for their bountiful blessings in the form of bumper harvest of agricultural crop, prosperity, protection of domesticated animals and humankind from epidemic diseases, protection of wildlife and biodiversity, and promotion of peace and social harmony in the society (Nabam 2013; Tag 2017).

\section{Ethnobotanical field survey and sample Size}

A brief ethnobotanical field survey was conducted in the Abotani biocultural landscape in 11 districts of Central Arunachal Pradesh for brief period of over 02 months during 2018 - 2019 following the quantitative ethnobotanical field method suggested by Martin (2008). Prior Inform Consent (PIC) was taken from the potential informants before start of the work. A total of 132 potential informants belonging to 66 household covering 22 villages were surveyed and interviewed from 11 districts. All the relevant ethnobotanical information including the vernacular name of plant species, part used, harvesting season and purpose of traditional uses shared by the potential informants were recorded in structured questionnaire format and field notebook. 
The herbarium specimen of each ethnobotanically significant species were collected and prepared following the method suggested by Jain \& Rao (1977). Each species were identified through consultation of standard regional flora such as Flora of Assam (Kanjilal et al. 1934 - 1940), The Flora of British India (Hooker 1875 - 1897), Flora of Lower Subansiri (Pal 1993), Materials for the Flora of Arunachal (Hajra et al. 1996; Giri et al. 2008; Chowdhery et al. 2009), e-Flora of China, and e-Herbarium of Kew. The accepted names were verified in the website www.theplantlist.org jointly hosted by Royal Botanic Garden, Kew UK and Missouri Botanical Garden, St. Louis USA, and Plants of World Online (POWO) hosted by RBG Kew. The taxonomically authenticated herbarium specimen bearing collection number and accession number were deposited in the Herbarium of Arunachal University (HAU), Department of Botany, Rajiv Gandhi University, Rono Hills, Doimukh, Arunachal Pradesh for future reference.

\section{RESULT \& DISCUSSION}

\section{Species diversity and habit}

Present cross cultural ethnobotanical investigations have revealed 45 ethnobotanically significant angiospermic plant species belonging to 34 genera clubbed within 27 families (Table 1; Figure 2) reported be used by the 05 tribes of Abotani Biocultural Landscape residing in 11 districts of Arunachal Pradesh. Among these, Asteraceae and Solanaceae were found to be most dominating group represented by highest number of 7 species each which is followed by Arecaceae, Cucurbitaceae and Poaceae represented by 2 species each (Figure 3). Among the 34 genera reported, the Solanum has been found to be most dominating genus represented by 5 species which is followed by Acmella, Artemisia, Capsicum and Cucumis represented by 2 species each (Figure 4). Herbs were represented by highest number of 23 species used by the Abotani tribes which is followed by shrubs ( 8 species), climbers (6), trees (5) and fern, grass, vine (1 each) (Figure 5).



Figure 2. Taxonomic diversity of significant ethnobotanical species (45 spp.) used by the Abotani Tribes of Arunachal Pradesh

\section{Diversity of ethnobotanical uses}

It has been revealed that the Abotani tribes of Arunachal Pradesh have rich ethnobotanical knowledge bases related to diverse use of plant resources available in their biocultural landscape. Of the 45-species recorded, 10 species are exclusively consumed as food, 24 species are used both as food and medicine, 4 species are exclusively used for medicinal purpose, 4 species are used in rituals and socio-cultural occasions, and 3 species are used for handicraft and construction purposes (Figures $6 \& 8$ ). 




Figure 3. Frequency of the most frequently used plant Families reported from Abotani Biocultural Landscape (ABL) in Arunachal Pradesh

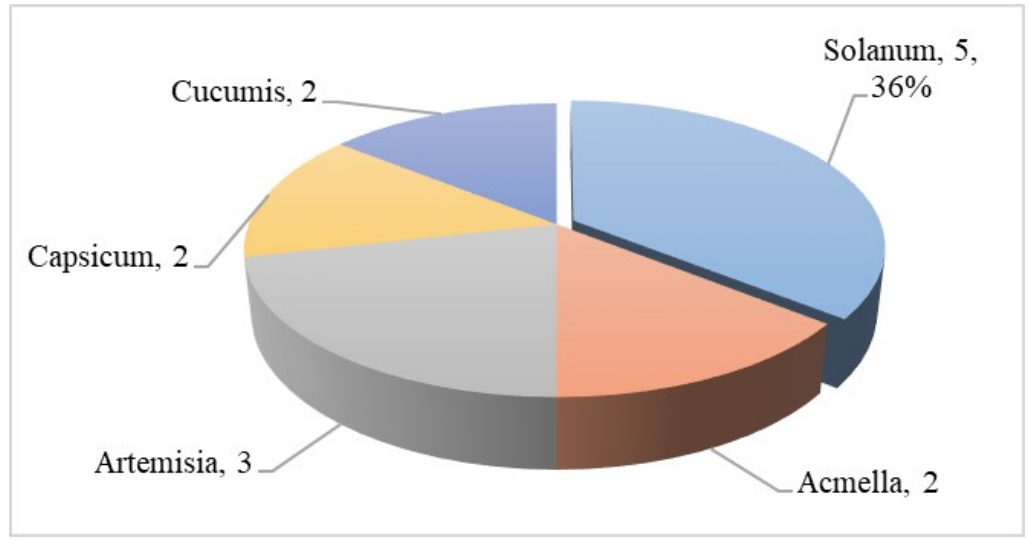

Figure 4. Statistics of frequently used genera



Figure 5. Diversity of habit and lifeform of significant ethnobotanical plants ( $45 \mathrm{spp}$.) reported from Abotani Biocultural Landscape (ABL) in Arunachal Pradesh 
Table 1. Checklist of cross cultural applications of significant ethnobotanical plants species used by the Abotani Tribes residing in 11 districts of Arunachal Pradesh, India

[Abbreviations used: $\mathrm{A}=$ Adi; $\mathrm{Ap}=$ Apatani; $\mathrm{G}=$ Galo; $\mathrm{T}=$ Tagin; $\mathrm{N}=$ Nyishi; $\mathrm{L}=$ Leaf; $\mathrm{F}=$ Fruit; WP $=$ Whole Plant; $\mathrm{R}=$ Rhizome; Tu = Tuber; $\mathrm{S}=$ Seed; $\mathrm{B}=$ Bark; YS = Young shoot; $\mathrm{Fr}=$ Frond]

\begin{tabular}{|c|c|c|c|c|c|}
\hline Botanical Names & $\begin{array}{l}\text { Vernacular } \\
\text { Name }\end{array}$ & Habits & $\begin{array}{l}\text { Part } \\
\text { used }\end{array}$ & $\begin{array}{c}\text { Type of } \\
\text { use }\end{array}$ & Ethnic use \\
\hline $\begin{array}{l}\text { Acmella oleracea (L.) R.K. } \\
\text { Jansen [Asteraceae]; } \\
\text { HY/HT/HAU/1604/2018 }\end{array}$ & $\begin{array}{l}\text { Marshang }(A), \\
\text { Yorkhung }(A p), \\
\text { Marsha }(G), \\
\text { Byadhi }(N), \\
\text { Marsha }(T)\end{array}$ & Herbs & $\mathrm{L}, \mathrm{F}$ & $\begin{array}{l}\text { Food, } \\
\text { medicine }\end{array}$ & $\begin{array}{l}\text { i. Flower and leaves are taken as } \\
\text { vegetable } \\
\text { ii. Remedy for cough } \\
\text { iii. Flower paste is applied in } \\
\text { toothache }\end{array}$ \\
\hline $\begin{array}{l}\text { Acmella paniculata (Wall. } \\
\text { ex DC.) R.K. Jansen } \\
\text { [Asteraceae]; } \\
\text { HY/HT/HAU/1608/2018 }\end{array}$ & $\begin{array}{l}\text { Marshang }(A), \\
\text { Yorkhung }(\text { Ap.), } \\
\text { Marsha }(G), \\
\text { Byadhi }(N), \\
\text { Marsha }(T)\end{array}$ & Herbs & WP & $\begin{array}{l}\text { Food, } \\
\text { Pesticide }\end{array}$ & $\begin{array}{l}\text { i. Leaves eaten raw and cooked with } \\
\text { other vegetable } \\
\text { ii. Whole plant paste is used as fish } \\
\text { poison }\end{array}$ \\
\hline $\begin{array}{l}\text { Acorus calamus } \mathrm{L} . \\
\text { [Acoraceae]; } \\
\text { HY/HT/HAU/1606/2018 }\end{array}$ & $\begin{array}{l}\text { Kile tolyo (Ap.), } \\
\text { Buch }(G), \text { Talyo } \\
(T)\end{array}$ & Herbs & $\mathrm{R}$ & Medicine & $\begin{array}{l}\text { i. Rhizome paste is applied on cuts, } \\
\text { wounds and bone fracture } \\
\text { ii. Extracts of rhizome along with } \\
\text { Zingiber officinalis rhizome is } \\
\text { used for curing Gastric } \\
\text { iii. Rhizome juice is used against } \\
\text { Asthma \& bronchitis. } \\
\text { iv. Aromatic oil is used for } \\
\text { treatment of joint pain, skin } \\
\text { inflammation, boil, cut and } \\
\text { wound. }\end{array}$ \\
\hline 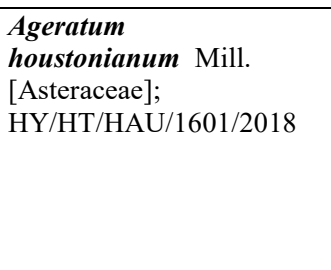 & $\begin{array}{l}\text { Namsing Ing/Elee } \\
(\text { A.), Borbia tami } \\
\text { (Ap.), Eae- } \\
\text { Namnya/Hiigo } \\
\text { remi }(G) \text { Pasho / } \\
\text { pasu-payou }(N), \\
\text { Garii / Myora }(T)\end{array}$ & Herbs & $\mathrm{L}$ & Medicine & $\begin{array}{l}\text { Paste is used for blood clotting and } \\
\text { wound healing }\end{array}$ \\
\hline $\begin{array}{l}\text { Allium hookeri Thwaites } \\
\text { [Amaryllidaceae]; } \\
\text { HY/HT/HAU/1603/2018 }\end{array}$ & $\begin{array}{l}\text { Diilap }(A), \text { Lepi } \\
\text { (Ap), Diilap } \\
(G), \text { Talap }(T) \text {, } \\
\text { Talap }(N)\end{array}$ & Herbs & $\begin{array}{l}\mathrm{Tu}, \\
\mathrm{L}\end{array}$ & $\begin{array}{l}\text { Food, } \\
\text { medicine }\end{array}$ & $\begin{array}{l}\text { i. Used as spice \& vegetable } \\
\text { ii. Used to cure Cold, cough \& } \\
\text { nausea }\end{array}$ \\
\hline $\begin{array}{l}\text { Alstonia scholaris R. Br. } \\
\text { [Apocynaceae]; } \\
\text { HT/HAU/1605/2019 }\end{array}$ & Popuk Siin (N) & Tree & $\mathrm{B}$ & Medicine & $\begin{array}{l}\text { Bark is used for treatment of sores } \\
\text { and soils. Pinch of powdered bark is } \\
\text { used for treatment of malarial fever. }\end{array}$ \\
\hline $\begin{array}{l}\text { Angiopteris } \\
\text { evecta (G.Forst.) Hoffm. } \\
\text { [Marattiaceae]; } \\
\text { HY/HT/HAU/1606/2018 }\end{array}$ & $\begin{array}{l}\text { Taba }(\text { A),Tari } \\
(\text { Ap), Bakum }(G), \\
\text { Nabay/Bom }(N) \\
\text { Tabii/Tach }(T)\end{array}$ & Shrub & $\mathrm{R}$ & $\begin{array}{l}\text { Famine } \\
\text { food, } \\
\text { medicine }\end{array}$ & $\begin{array}{l}\text { i. Roots are made into paste and } \\
\text { shed dried for 3-4 days and } \\
\text { packed on leaves and cooked. } \\
\text { ii. Anti-diarrhoeic and Anti- } \\
\text { dysentery }\end{array}$ \\
\hline $\begin{array}{l}\text { Artemisia indica Willd. } \\
\text { [Asteraceae]; } \\
\text { HY/HT/HAU/1608/2018 }\end{array}$ & $\begin{array}{l}\text { Kuklyu }(\text { Ap.), } \\
\text { Tape-Nyobeng } \\
\text { (G), Tapin }(N) \text {, } \\
\text { Tappen }(T),\end{array}$ & Shrubs & $\mathrm{L}$ & $\begin{array}{l}\text { Food, } \\
\text { medicine }\end{array}$ & $\begin{array}{l}\text { i. Vegetable } \\
\text { ii. Leaves are kept in poultry } \\
\text { baskets to repel pests and insects. } \\
\text { iii. Inhaling strong smell of leaf } \\
\text { relieves nose blockade and } \\
\text { headache } \\
\text { iv. Bathing with water diluted with } \\
\text { its leaves relieves from itching \& } \\
\text { skin allergy } \\
\text { v. Fresh juice from leaves is used } \\
\text { for curing eye redness }\end{array}$ \\
\hline
\end{tabular}


Hage Yanka et al. 275

\begin{tabular}{|c|c|c|c|c|c|}
\hline Botanical Names & $\begin{array}{l}\text { Vernacular } \\
\text { Name }\end{array}$ & Habits & $\begin{array}{l}\text { Part } \\
\text { used }\end{array}$ & $\begin{array}{l}\text { Type of } \\
\text { use }\end{array}$ & Ethnic use \\
\hline $\begin{array}{l}\text { Artemisia nilagirica } \\
\text { (C.B.Clarke) Pamp. } \\
\text { [Asteraceae] } \\
\text { HY/HT/HAU/1610/2018 }\end{array}$ & $\begin{array}{l}\text { Kuklyu }(\text { Ap), } \\
\text { Tappen }(T), \\
\text { Tape-Nyobeng } \\
(G), \text { Tapin }(N)\end{array}$ & Shrubs & $\mathrm{L}$ & $\begin{array}{l}\text { Food, } \\
\text { medicine }\end{array}$ & $\begin{array}{l}\text { i. Leaves used in stomach-ache and } \\
\text { body pain. } \\
\text { ii. Young aromatic leaves used as } \\
\text { disinfectant }\end{array}$ \\
\hline $\begin{array}{l}\text { Begonia roxburghii (Miq.) } \\
\text { A.DC. [Begoniaceae] } \\
\text { HY/HT/HAU/1612/2018 }\end{array}$ & $\begin{array}{l}\text { Sisi-Baying }(A), \\
\text { Lukhu }(\text { Ap), } \\
\text { Buku-Hurbu }(G), \\
\text { Bakku yulu (T), } \\
\text { Biku Yulu (N) }\end{array}$ & Herb & $\mathrm{S}, \mathrm{R}$ & $\begin{array}{l}\text { Food, } \\
\text { medicine }\end{array}$ & $\begin{array}{l}\text { i. The stem is peeled and eaten } \\
\text { ii. Root paste is used as a } \\
\text { deworming agent }\end{array}$ \\
\hline $\begin{array}{l}\text { Brassica juncea (L.) Czern. } \\
\text { [Brassicaceae]; } \\
\text { HY/HT/HAU/1615/2018 }\end{array}$ & $\begin{array}{l}\text { Pattu oin }(A), \\
\text { Giyang Hamang } \\
\text { (Ap.), Giiyi }(G), \\
\text { Goying } / \text { Goi }(T) \text {, } \\
\text { Guyi } O(N) \text {, }\end{array}$ & Herb & $\mathrm{L}$ & Food & The leaves are used as vegetable \\
\hline $\begin{array}{l}\text { Calamus acanthospathus } \\
\text { Griff. [Arecaceae]; } \\
\text { HY/HT/HAU/1620/2018 }\end{array}$ & Taser (Ap) & Climber & WP & $\begin{array}{l}\text { Construc } \\
\text { tion, } \\
\text { rituals, } \\
\text { crafts }\end{array}$ & $\begin{array}{l}\text { i. House construction } \\
\text { ii. Making accessories in Rituals } \\
\text { and festivals } \\
\text { iii. For crafting of traditional } \\
\text { Baskets }\end{array}$ \\
\hline $\begin{array}{l}\text { Calamus leptospadix Griff. } \\
\text { [Arecaceae]; } \\
\text { HY/HT/HAU/1616/2018 }\end{array}$ & $\operatorname{Tabh}(G)$ & Climber & WP & $\begin{array}{l}\text { Constru- } \\
\text { ction, } \\
\text { rituals, } \\
\text { crafts }\end{array}$ & $\begin{array}{l}\text { Used as roof for binding in house } \\
\text { construction }\end{array}$ \\
\hline $\begin{array}{l}\text { Capsicum annum } \mathrm{L} . \\
\text { [Solanaceae]; } \\
\text { HY/HT/HAU/1618/2018 }\end{array}$ & $\begin{array}{l}\text { Sibol (A), Yobbu } \\
\text { Terro (Ap),Yaluk } \\
\text { (G), Yaluk }(G), \\
\text { Hilup (N) }\end{array}$ & Herb & $\mathrm{F}$ & $\begin{array}{l}\text { Food, } \\
\text { medicine }\end{array}$ & $\begin{array}{l}\text { i. Important part of traditional food } \\
\text { habit; it is taken raw, boil, as } \\
\text { chutney and condiment } \\
\text { ii. Taken to cure fever, cold and } \\
\text { cough }\end{array}$ \\
\hline $\begin{array}{l}\text { Capsicum chinense Jacq. } \\
\text { [Solanaceae]; } \\
\text { HY/HT/HAU/1622/2018 }\end{array}$ & $\begin{array}{l}\text { Maan mirsi/ } \\
\text { Sibol mirsi/ } \\
\text { Sibor mirsi (A), } \\
\text { Tagin Terro } \\
\text { (Ap), Nyipak } \\
\text { mircha (T), } \\
\text { Bongar Hilup } \\
\text { (N) }\end{array}$ & Herb & $\mathrm{F}$ & $\begin{array}{l}\text { Food, } \\
\text { medicine }\end{array}$ & $\begin{array}{l}\text { i. It is taken raw and boil as chutney } \\
\text { and condiments } \\
\text { ii. Taken to cure fever, cold and } \\
\text { cough }\end{array}$ \\
\hline $\begin{array}{l}\text { Clerodendrum } \\
\text { glandulosum } \text { Lindl. } \\
\text { [Lamiaceae]; } \\
\text { HY/HT/HAU/1623/2018 }\end{array}$ & $\begin{array}{l}\text { Ongin (A), Pato } \\
\text { Hamang (Ap), } \\
\text { Oin }(\mathrm{G}), \text { Tiipin } \\
\text { potho }(\mathrm{N})\end{array}$ & Shrub & $\mathrm{L}$ & $\begin{array}{l}\text { Food, } \\
\text { medicine }\end{array}$ & $\begin{array}{l}\text { i. Decoction of leaves is used for } \\
\text { lowering high Blood pressure } \\
\text { ii. Consumed as vegetable }\end{array}$ \\
\hline $\begin{array}{l}\text { Crassocephalum } \\
\text { crepidiodes (Benth.) } \\
\text { S.Moore [Asteraceae]; } \\
\text { HY/HT/HAU/1625/2018 }\end{array}$ & $\begin{array}{l}\text { Gende/Ibel }(A) \\
\text { Genda Hamang } \\
(\text { Ap), Iing- } \\
\text { Kayeng }(T), \\
\text { Gendu }(G), \\
\text { Yamin } O(N)\end{array}$ & Herb & WP & $\begin{array}{l}\text { Food, } \\
\text { medicine }\end{array}$ & $\begin{array}{l}\text { i. Leaf paste is used to cure cuts and } \\
\text { wounds } \\
\text { ii. Decoction of the plant is used to } \\
\text { cure insomnia } \\
\text { iii. Whole plant is used as a } \\
\text { vegetable }\end{array}$ \\
\hline $\begin{array}{l}\text { Cucumis melo } \mathrm{L} . \\
\text { [Cucurbitaceae]; } \\
\text { HY/HT/HAU/1621/2018 }\end{array}$ & $\begin{array}{l}\text { Asi Tapa }(A), \\
\text { Tape Hamang } \\
\text { (Ap), Patum }(G) \text {, } \\
\text { Tapẽh }(T) \text { Tapeh } \\
\text { (N) }\end{array}$ & Climber & $\mathrm{L}, \mathrm{F}$ & Food & $\begin{array}{l}\text { i. Leaves taken as vegetable } \\
\text { ii. Fruits are cooked and consumed }\end{array}$ \\
\hline
\end{tabular}




\begin{tabular}{|c|c|c|c|c|c|}
\hline Botanical Names & $\begin{array}{l}\text { Vernacular } \\
\text { Name }\end{array}$ & Habits & $\begin{array}{l}\text { Part } \\
\text { used }\end{array}$ & $\begin{array}{l}\text { Type of } \\
\text { use }\end{array}$ & Ethnic use \\
\hline $\begin{array}{l}\text { Cucumis sativus L. } \\
\text { [Cucurbitaceae]; } \\
\text { HY/HT/HAU/1622/2018 }\end{array}$ & $\begin{array}{l}\text { Makung (A), } \\
\text { Taku (Ap), Meku } \\
(G), M e k u(T), \\
\text { Muku (N) }\end{array}$ & Climber & $\mathrm{F}$ & Food & Fruit is eaten raw as a salad \\
\hline $\begin{array}{l}\text { Dendrocalamus } \\
\text { hamiltonii Nees \& Arn. ex } \\
\text { Munro [Poaceae]; } \\
\text { HY/HT/HAU/1624/2018 }\end{array}$ & $\begin{array}{l}\text { Ea (A), Yai } \\
\text { byapu (Ap), Eh } \\
\text { Henu (T), Diba } \\
(G), \text { Hej (N) }\end{array}$ & Grass & $\begin{array}{l}\mathrm{S} \\
\mathrm{YS}\end{array}$ & $\begin{array}{l}\text { Food, } \\
\text { rituals }\end{array}$ & $\begin{array}{l}\text { i. Young shoots are used as } \\
\text { vegetable } \\
\text { ii. Shoots fermented are used to } \\
\text { prepare ikhu/Hikhu/Hiyup } \\
\text { iii. Stem is also used in marriage } \\
\text { ceremony and customary } \\
\text { Rituals }\end{array}$ \\
\hline $\begin{array}{l}\text { Dioscorea bulbifera } \mathrm{L} . \\
\text { [Dioscoreaceae]; } \\
\text { HY/HT/HAU/1627/2019 }\end{array}$ & $\begin{array}{l}\text { Egin }(A), \text { Huli- } \\
\text { Hula }(\text { Ap), Egin } \\
\text { Tabi/Ejo }(G), \\
\text { Hiili }(T), \text { Hes }(N)\end{array}$ & Climber & $\begin{array}{l}\mathrm{B}, \\
\mathrm{L}\end{array}$ & $\begin{array}{l}\text { Food, } \\
\text { fodder }\end{array}$ & $\begin{array}{l}\text { i. Bulb are either roasted or boiled } \\
\text { and taken as food and vegetable. } \\
\text { ii. Leaves are used as fodder }\end{array}$ \\
\hline $\begin{array}{l}\text { Diplazium esculentum } \\
\text { (Retz.) Sw. [Athyriaceae]; } \\
\text { HY/HT/HAU/1630/2019 }\end{array}$ & $\begin{array}{l}\text { Takang }(A), \\
\text { Hiika Hamang } \\
(\text { Ap), Taka }(G), \\
\text { Pakya-Raya }(T) \\
\text { Taka paya }(N)\end{array}$ & Fern & $\mathrm{Fr}$ & Food & $\begin{array}{l}\text { The tender fronds are boiled and } \\
\text { used as a vegetable }\end{array}$ \\
\hline $\begin{array}{l}\text { Eryngium foetidum } \mathrm{L} . \\
\text { [Apiaceae]; } \\
\text { HY/HT/HAU/1632/2019 }\end{array}$ & $\begin{array}{l}\text { Ori, Ori-ritak, } \\
\text { migom- ori }(A) \text {, } \\
\text { dhuniyapatta } \\
(\text { Ap), Rithak }(G) \text {, } \\
\text { Nyipak Dhania } \\
(T), \text { Jongli } \\
\text { Dhaniya }(N)\end{array}$ & Herbs & $\mathrm{L}$ & Food & $\begin{array}{l}\text { Leaves used in chutney, salad and } \\
\text { boiled food }\end{array}$ \\
\hline $\begin{array}{l}\text { Glycine max (L.) Merr. } \\
\text { [Fabaceae]; } \\
\text { HY/HT/HAU/1636/2019 }\end{array}$ & $\begin{array}{l}\text { Rontung }(\mathrm{A}), \\
\text { Potung perung } \\
(\text { Ap), Yagya }(\mathrm{G}), \\
\text { Peren }(\mathrm{N})\end{array}$ & Shrubs & $\mathrm{S}$ & Food & $\begin{array}{l}\text { Seeds are consumed after boiling } \\
\text { and fermented seeds are eaten } \\
\text { chutney. }\end{array}$ \\
\hline $\begin{array}{l}\text { Houttuynia cordata Thunb. } \\
\text { [Saururaceae]; } \\
\text { HY/HT/HAU/1634/2019 }\end{array}$ & $\begin{array}{l}\text { Roram, Reram } \\
\text { (A), Siiya } \\
\text { Hamang (Ap), } \\
\text { Moyumkneme } \\
\text { (G), Hiingya }(T) \\
\text { Hongyea/ Tadar } \\
\text { O/Hiiya }(N)\end{array}$ & Herbs & WP & $\begin{array}{l}\text { Food, } \\
\text { medicine }\end{array}$ & $\begin{array}{l}\text { i. Eaten raw for indigestion, and } \\
\text { Chutney making. } \\
\text { ii. Use for treating chest, lung and } \\
\text { skin inflammation, and stomach } \\
\text { ulcer. }\end{array}$ \\
\hline $\begin{array}{l}\text { Impatiens racemosa } \mathrm{DC} . \\
\text { [Balsaminaceae]; } \\
\mathrm{HY} / \mathrm{HT} / \mathrm{HAU} / 1659 / 2019\end{array}$ & $\begin{array}{l}\text { Nanor tangkor } \\
(\text { A), Aki tai } \\
\text { Hamang }(\text { Ap.), } \\
\text { Nyamchi }(G) \text {, } \\
\text { Nyamche }(T) \text {, } \\
\text { Choyom }(N)\end{array}$ & Herb & $\mathrm{L}$ & Food & $\begin{array}{l}\text { Leaves are boiled and consumed as } \\
\text { vegetable }\end{array}$ \\
\hline $\begin{array}{l}\text { Ipomoea batatas (L.) Lam. } \\
\text { [Convolvulaceae]; } \\
\text { HY/HT/HAU/1637/2019 }\end{array}$ & $\begin{array}{l}\text { Egin tarii }(A), \\
\text { Mita Alu }(\text { Ap. }), \\
\text { Ramya/Karya- } \\
\text { Riyamiya }(T), \\
\text { Egin Pagre }(N)\end{array}$ & Vine & $\mathrm{Tu}$ & Food & $\begin{array}{l}\text { Tubers are either roasted or boiled } \\
\text { and taken as food }\end{array}$ \\
\hline $\begin{array}{l}\text { Litsea cubeba (Lour.) Pers. } \\
\text { [Lauraceae]; } \\
\text { HY/HT/HAU/1639/2019 }\end{array}$ & $\begin{array}{l}\text { Tayer Rajil } \\
\text { /Taier /rayil (A), } \\
\text { Santero }(\text { Ap) }\end{array}$ & Tree & $\mathrm{F}$ & $\begin{array}{l}\text { Food, } \\
\text { medicine }\end{array}$ & $\begin{array}{l}\text { i. Fruit is used as a condiments in } \\
\text { curries and chutney } \\
\text { ii. Fruits eaten as a remedy to cold } \\
\text { and cough. }\end{array}$ \\
\hline
\end{tabular}




\begin{tabular}{|c|c|c|c|c|c|}
\hline Botanical Names & $\begin{array}{l}\text { Vernacular } \\
\text { Name }\end{array}$ & Habits & $\begin{array}{l}\text { Part } \\
\text { used }\end{array}$ & $\begin{array}{l}\text { Type of } \\
\text { use }\end{array}$ & Ethnic use \\
\hline Litsea cubeba (contd.) & $\begin{array}{l}\text { Tayer/Ischi } \\
\text { takke ame }(G), \\
\text { Tayir }(T), \text { Sin-ter } \\
(N)\end{array}$ & & & & $\begin{array}{l}\text { iii. Oil is used for curing joint and } \\
\text { muscle pain, relieve tissue } \\
\text { inflammation, high blood } \\
\text { pressure and insomnia. }\end{array}$ \\
\hline $\begin{array}{l}\text { Mikania micrantha Kunth } \\
\text { [Asteraceae]; } \\
\text { HY/HT/HAU/1644/2019 }\end{array}$ & $\begin{array}{l}\text { Sina lota/ Mali } \\
\text { Riimang }(A), \\
\text { Manii Tami }(A p) \text {, } \\
\text { Lindetare }(G) \text {, } \\
\text { Gandi Ter }(N)\end{array}$ & Climber & $\mathrm{L}$ & Medicine & $\begin{array}{l}\text { Paste of leaves and stem is used for } \\
\text { healing wounds or clotting of blood. }\end{array}$ \\
\hline $\begin{array}{l}\text { Panax arunachalensis } \\
\text { M.Taram, A.P. Das \& } \\
\text { H.Tag [Araliaceae]; } \\
\text { MT/HT/1505/2018 }\end{array}$ & $\begin{array}{l}\text { Mud Pigri }(\mathrm{N}) \\
\text { Zansang }(\mathrm{Ap}) \\
\text { Gainsing }(\mathrm{A}) \\
\text { Pagru }(\mathrm{T}) \\
\text { Miitum angina }(\mathrm{G})\end{array}$ & Herb & $\mathrm{R}$ & Medicine & $\begin{array}{l}\text { Powdered rhizome are used for } \\
\text { treatment of chest inflammation, } \\
\text { lung inflammation, fatigue, boil and } \\
\text { sores, mouth ulcer and apply in cut } \\
\text { wound. }\end{array}$ \\
\hline $\begin{array}{l}\text { Panax bipinnatifidus Seem. } \\
\text { [Araliaceae]; } \\
\text { HT/SK/HAU/1542/2017 }\end{array}$ & $\begin{array}{l}\text { Mud Siw Pigri } \\
\text { (N), Zansang } \\
\text { (Ap), Gainsing } \\
\text { (A), Pagru (T), } \\
\text { Miitum angina } \\
\text { (G) }\end{array}$ & Herb & $\mathrm{R}$ & $\begin{array}{l}\text { Food, } \\
\text { medicine }\end{array}$ & $\begin{array}{l}\text { Rhizome are used as herbal drink as } \\
\text { health tonic. Dried rhizome powder } \\
\text { are used for treatment of stomach } \\
\text { ulcer, boil and sores, mouth ulcer } \\
\text { chest inflammation. }\end{array}$ \\
\hline $\begin{array}{l}\text { Panax bipinnatifidus var. } \boldsymbol{a} \\
\text { ngustifolius (Burkill) J.Wen } \\
\text { [Araliaceae]; } \\
\text { HT/SK/HAU/1597/2018 }\end{array}$ & $\begin{array}{l}\text { Mud Siw Pigri } \\
\text { (N), Zansang } \\
\text { (Ap), Gainsing } \\
\text { (A), Pagru (T) } \\
\text { Miitum angina } \\
\text { (G) }\end{array}$ & Herb & $\mathrm{R}$ & $\begin{array}{l}\text { Food, } \\
\text { medicine }\end{array}$ & $\begin{array}{l}\text { Ripen fruits eaten raw. Powder of } \\
\text { slice cut rhizome are used as herbal } \\
\text { tea to boost stamina. Pinch of } \\
\text { powder are used for treatment of } \\
\text { chest inflammation, boil and sores, } \\
\text { mouth ulcer. }\end{array}$ \\
\hline $\begin{array}{l}\text { Paris polyphylla } \mathrm{Sm} . \\
\text { [Melanthiaceae]; } \\
\text { HT/HAU/1557/2017 }\end{array}$ & $\begin{array}{l}\text { Mutum Enge, } \\
\text { Tabu Billo }(\mathrm{N}), \\
\text { Nyomrang } \\
\text { Takeng, Orpo \& } \\
\text { Kangkom Oying } \\
\text { (A) }\end{array}$ & Herb & $\mathrm{R}$ & $\begin{array}{l}\text { Food, } \\
\text { medicine }\end{array}$ & $\begin{array}{l}\text { The leaves and fruits are edible. The } \\
\text { smoked dried tuber is used for } \\
\text { treatment of debility and boil and } \\
\text { sores, skin inflammation, chest } \\
\text { inflammation. }\end{array}$ \\
\hline $\begin{array}{l}\text { Phoebe cooperiana } \\
\text { P.C.Kanjilal \& Das } \\
\text { [Lauraceae]; } \\
\text { HY/HT/HAU/1642/2019 }\end{array}$ & $\begin{array}{l}\text { Tapir }(A), \text { Hisir } \\
(G), \text { Samper }(A p), \\
\text { Sechar }(T), \text { Jishir } \\
(N)\end{array}$ & Tree & $\mathrm{F}$ & $\begin{array}{l}\text { Food, } \\
\text { medicine }\end{array}$ & $\begin{array}{l}\text { i. Fruit eaten raw as a Chutney } \\
\text { ii. Fruit also boiled as food and to } \\
\text { improve liver function, relieve } \\
\text { insomnia and high blood pressure. } \\
\text { iii. Pulp applied on inflamed burnt } \\
\text { skin as anti-inflammatory agent }\end{array}$ \\
\hline $\begin{array}{l}\text { Piper pedicellatum } \text { C.DC. } \\
\text { [Piperaceae]; } \\
\text { HY/HT/HAU/1646/2019 }\end{array}$ & $\begin{array}{l}\text { Lorii }(A), \text { Rarel } \\
\text { Raru }(\text { Ap. }), \text { Rer } \\
(G), \text { Yarii }(T), \\
\text { Raar/Ler } O(N)\end{array}$ & Shrub & $\mathrm{L}$ & $\begin{array}{l}\text { Food, } \\
\text { medicine }\end{array}$ & $\begin{array}{l}\text { i. Consumed as vegetable } \\
\text { ii. It is also consumed to relieve } \\
\text { high blood pressure. }\end{array}$ \\
\hline $\begin{array}{l}\text { Rubus ellipticus } \mathrm{Sm} . \\
\text { [Rosaceae]; } \\
\text { HY/HT/HAU/1647/2019 }\end{array}$ & $\begin{array}{l}\text { Pakkom Tayin } \\
\text { (A), Jiling ayi / } \\
\text { Jilyun (Ap), Tae } \\
\text { (T) }\end{array}$ & Shrub & $\mathrm{F}$ & Food & Fruit is eaten raw \\
\hline $\begin{array}{l}\text { Saccharum spontaneum } \text { L. } \\
\text { [Poaceae]; } \\
\text { HY/HT/HAU/1642/2019 }\end{array}$ & $\begin{array}{l}\text { Tapi (A), Peji } \\
\text { Paelo (Ap) }\end{array}$ & Shrubs & WP & $\begin{array}{l}\text { Ritual, } \\
\text { construct } \\
\text { ion }\end{array}$ & $\begin{array}{l}\text { i. Whole plant is used in different } \\
\text { rituals and used to cure insomnia } \\
\text { ii. House construction } \\
\text { iii. Mat making }\end{array}$ \\
\hline $\begin{array}{l}\text { Saurauia punduana Wall. } \\
\text { [Actinidiaceae]; } \\
\text { HY/HT/HAU/1649/2019 }\end{array}$ & $\begin{array}{l}\text { Taan (A), Enchi } \\
\text { (G), Hinchi (T), } \\
\text { Nyinch (N) }\end{array}$ & Tree & WP & Ritual & $\begin{array}{l}\text { Whole plant are considered to be } \\
\text { sacred and is used in rituals. Berry } \\
\text { are edible. }\end{array}$ \\
\hline
\end{tabular}


278 Cross-cultural ethnobotany of Abotani tribes

\begin{tabular}{|c|c|c|c|c|c|}
\hline Botanical Names & $\begin{array}{l}\text { Vernacular } \\
\text { Name }\end{array}$ & Habits & $\begin{array}{l}\text { Part } \\
\text { used }\end{array}$ & $\begin{array}{l}\text { Type of } \\
\text { use }\end{array}$ & Ethnic use \\
\hline $\begin{array}{l}\text { Solanum aethiopicum } \mathrm{L} . \\
\text { [Solanaceae]; } \\
\text { HY/HT/HAU/1653/2019 }\end{array}$ & $\begin{array}{l}\text { Kopii }(A), \text { Byako } \\
(\text { Ap), Kope }(G), \\
\text { Kesia Bake }(T), \\
\text { Byak }(N)\end{array}$ & Herb & $\mathrm{F}$ & $\begin{array}{l}\text { Food, } \\
\text { medicine }\end{array}$ & $\begin{array}{l}\text { Fruits are boiled and consumed as } \\
\text { Chutney or vegetable }\end{array}$ \\
\hline $\begin{array}{l}\text { Solanum americanum Mill. } \\
\text { [Solanaceae]; } \\
\text { HY/HT/HAU/1656/2019 }\end{array}$ & $\begin{array}{l}\text { Okomamang }(A), \\
\text { Hiiro Hamang } \\
(A p), \text { Or-Re }(G) \\
\text { Hor } O(T \& N)\end{array}$ & Herb & $\mathrm{L}, \mathrm{F}$ & $\begin{array}{l}\text { Food, } \\
\text { medicine }\end{array}$ & $\begin{array}{l}\text { i. Leaves are boiled and consumed } \\
\text { as vegetable and also used for } \\
\text { curing Diabetes, stomach pain } \\
\text { and cough } \\
\text { ii. Ripe fruit are eaten raw }\end{array}$ \\
\hline $\begin{array}{l}\text { Solanum torvum } \mathrm{Sw} . \\
\text { [Solanaceae]; } \\
\text { HY/HT/HAU/1654/2016 }\end{array}$ & $\begin{array}{l}\text { Kodu/Kopii } \\
\text { Piiro }(A), \\
\text { Mishang Byako } \\
\text { (Ap), Baak }(G), \\
\text { Sete Bake }(T), \\
\text { Pata Byak }(N)\end{array}$ & Herb & $\mathrm{F}$ & $\begin{array}{l}\text { Food, } \\
\text { medicine }\end{array}$ & $\begin{array}{l}\text { i. Fruits are cooked and made into } \\
\text { paste and consumed as Chutney } \\
\text { ii. Boiled fruits are used against } \\
\text { diabetes and stomach disorder }\end{array}$ \\
\hline $\begin{array}{l}\text { Solanum tuberosum L. } \\
\text { [Solanaceae]; } \\
\text { HY/HT/HAU/1652/2019 }\end{array}$ & $\begin{array}{l}\text { Aalu }(A, A p ., G, \\
T, N)\end{array}$ & Herb & $\mathrm{T}$ & Food & $\begin{array}{l}\text { Tubers are either roasted, boiled or } \\
\text { fried and taken as food }\end{array}$ \\
\hline $\begin{array}{l}\text { Solanum anguivi Lam } \\
\text { [Solanaceae]; } \\
\text { HY/HT/HAU/1657/2019 }\end{array}$ & $\begin{array}{l}\text { Kopii Piimiik } \\
\text { (A), Adi Byako } \\
\text { (Ap), Adi Baak } \\
(G), \text { Adi Baake } \\
(T), \text { Adi Byak (N) }\end{array}$ & Herb & $\mathrm{F}$ & $\begin{array}{l}\text { Food, } \\
\text { medicine }\end{array}$ & $\begin{array}{l}\text { i. Fruits are boiled and made into } \\
\text { paste and consumed as Chutney } \\
\text { ii. Boiled fruit used against diabetes } \\
\text { and stomach disorder }\end{array}$ \\
\hline $\begin{array}{l}\text { Zanthoxylum rhetsa } \\
\text { (Roxb.) DC. [Rutaceae]; } \\
\text { HY/HT/HAU/1661/2019 }\end{array}$ & $\begin{array}{l}\text { Ombe or ombeng, } \\
\text { Ongar }(A), \\
\text { Yakhung }(A p), \\
\text { Hiibe/ Onyor }(G) \text {, } \\
\text { Honyor }(T), \\
\text { Honyir }(N)\end{array}$ & Tree & $\mathrm{L}, \mathrm{F}$ & $\begin{array}{l}\text { Food, } \\
\text { medicine }\end{array}$ & $\begin{array}{l}\text { i. Leaf are boiled and consume as } \\
\text { vegetable and seasoning } \\
\text { ii. Fruits are used for fish poisoning }\end{array}$ \\
\hline $\begin{array}{l}\text { Zingiber officinale } \text { Roscoe } \\
\text { [Zingiberaceae]; } \\
\text { HY/HT/HAU/1533/2018 }\end{array}$ & $\begin{array}{l}\text { Takeng (A), Taki } \\
(\text { Ap), Takkeh } \\
(G), \text { Tak-Kesi } \\
(T), \text { Takq }(N) \\
\end{array}$ & Herb & $\mathrm{R}$ & $\begin{array}{l}\text { Food, } \\
\text { medicine }\end{array}$ & $\begin{array}{l}\text { i. Used as a condiment } \\
\text { ii. Taken raw to cure fever, } \\
\text { headache, cold and cough }\end{array}$ \\
\hline
\end{tabular}

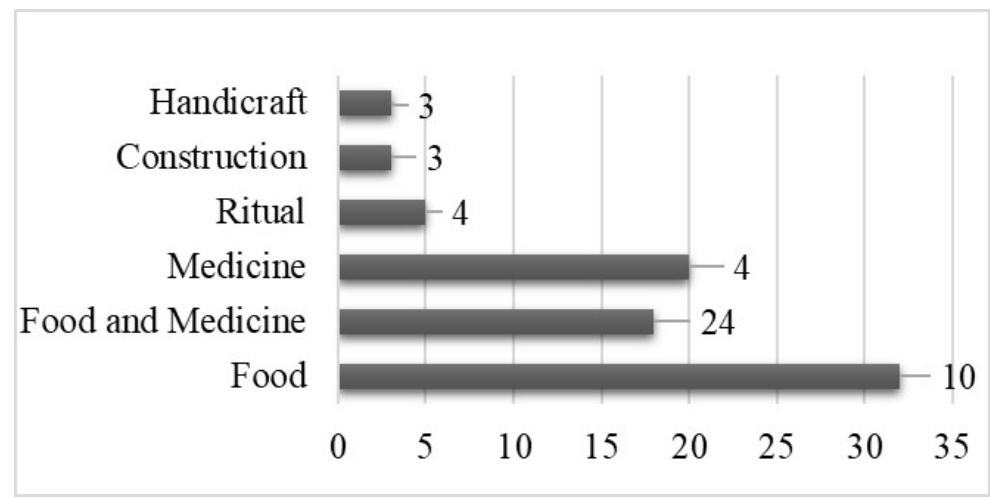

Figure 6. Diversity of ethnobotanical uses of plants (45 spp.) in Abotani Biocultural Landscape (ABL) in Arunachal Pradesh

\section{Harvesting and utilization of plants parts}

Of the total 45 species of angiosperm reported from the Abotani Biocultural Landscape (ABL), it has been observed that the leaves were most frequently harvested plant parts (15 spp.), while fruits are harvested from 13 species, whole plant used are harvested from 7 

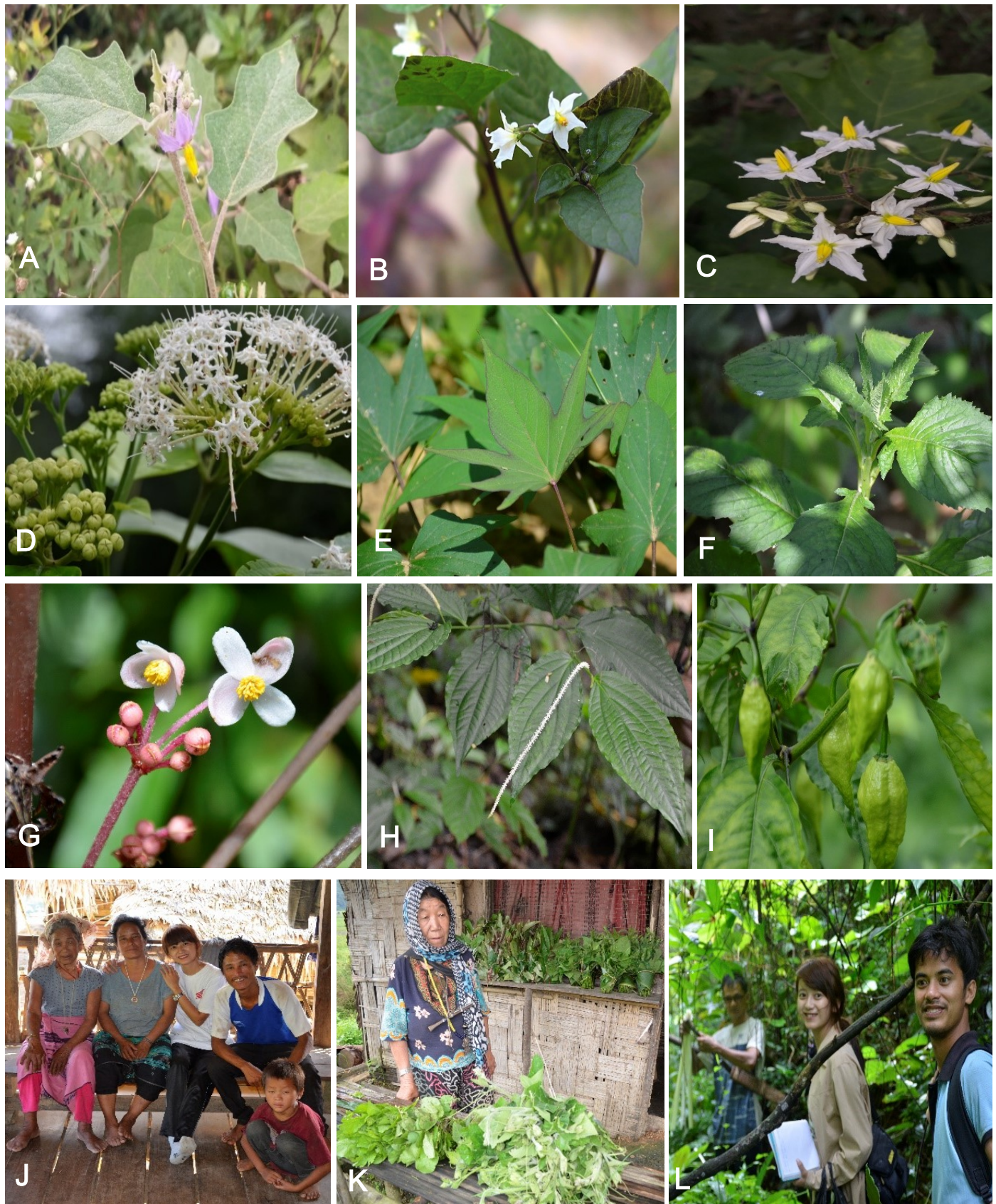

PLATE - I. Some significant ethnobotanical plants of Abotani tribes of Arunachal Pradesh: A. Solanum anguivi; B. Solanum americanum; C. Solanum torvum; D. Clerodendrum glandulosum; E. Ipomoea batatas; F. Crassocephalum crepidiodes; G. Begonia roxburghii; H. Piper pedicellatum; I. Capsicum chinence; J. Interaction with local informants; K. Local tribal women selling organic vegetable; L. Collection of ethnobotanical plants in the field with potential local informants. 
species, rhizome part used are harvested from 7 species, tuber and seed parts used are harvested from 3 species, and bark ( 2 spp.), frond and young shoot parts used are harvested from single species (Figure 7).

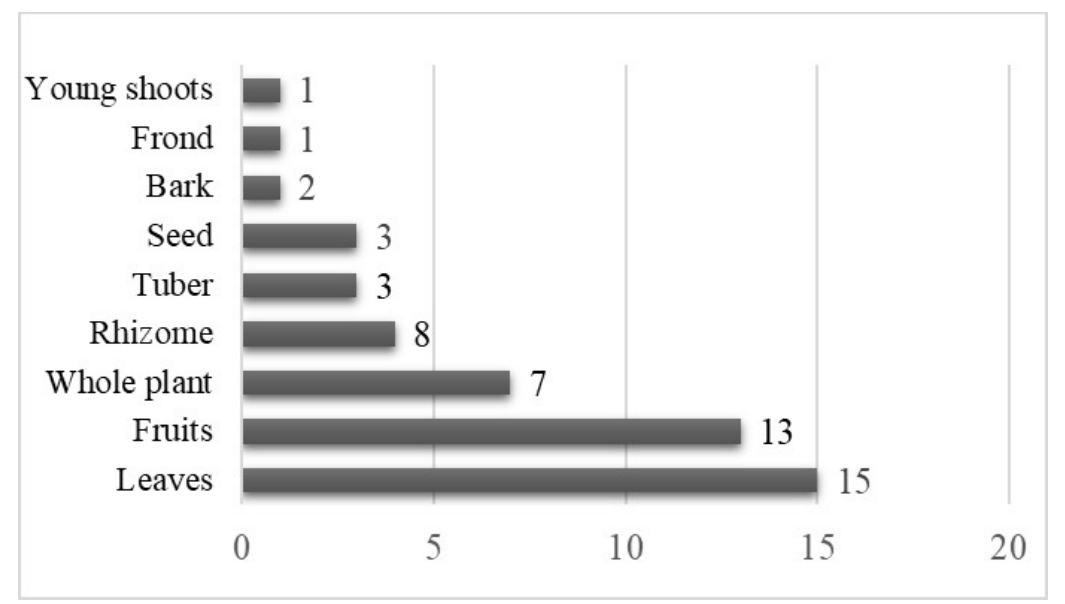

Figure 7. Diversity of plant parts harvested from significant ethnobotanical species of the Abotani Biocultural Landscape (ABL) in Arunachal Pradesh



Figure 8. Cross use of 38 species (out of total $45 \mathrm{spp}$.) of food and medicinal plants reported from the Abotani Biocultural Landscape in Arunachal Pradesh

\section{Ethnopharmacological uses}

Of the 45 ethnobotanically useful plant species reported, almost 24 species (50\%) are found to be used in traditional folk medicine for treatment of different ailments and diseases such as Diabetes, fever, stomach disorder, cold, cough, wounds, cuts etc. Majority of the species reported are used as both food and medicinal agents whereas only 04 species are exclusively used as medicine and 10 species are exclusively used as food (Fig.8). Some of the ethnopharmacologically significant species reported from the Abotani Biocultural Landscapes (ABL) are Acorus calamus, Ageratum houstonianum, Allium hookeri, Alstonia scholaris, Angiopteris evecta, Artemisia indica, Artemisia nilagirica, Begonia roxburghii, Houttuynia cordata, Litsea cubeba, Mikania micrantha, Paris polyphylla, Panax bipinnatifidus, Panax bipinnatifidus var. angustifolius, Panax arunachalensis, Phoebe cooperiana and Zanthoxylum rhetsa.

\section{Species used for local food security}

The five tribes, namely, Nyishi, Adi, Galo, Apatani and Tagin residing in the Abotani Biocultural Landscapes (ABL) of Arunachal Pradesh are solely dependent on natural resources from community forest land and agricultural land for the food and livelihood security. 
Of the 45 angiosperm species reported from their respective biocultural landscape, the majority of $32(80 \%)$ species reported are used for ensuring local food security. These species are either cultivated or harvested from the wild, and the most frequently used species are cultivated in traditional home garden and jhum plots whereas the wild edible vegetables and tubers are occasionally harvested from the community forest land as supplementary food items. The nutritionally and commercially viable local food plants reported to be available in the traditional biocultural landscape of Abotani tribes are Piper pedicellatum, Solanum aethiopicum, Solanum americanum, Solanum torvum, Solanum violaceum, Solanum tuberosum, Zingiber officinale, Acmella oleracea, Acmella paniculata, Capsicum annum, Capsicum annum hybrid, Capsicum frutescens, Clerodendrum glandulosum, Crassocephalum crepidiodes, Houttuynia cordata, Litsea cubeba, Zanthoxylum rhetsa, Brassica juncea, Cucumis melo, Cucumis sativus, Eryngium foetidum, Glycine max, Begonia roxburghii, and Dendrocalamus hamiltonii.

\section{Species used in local rituals and other purposes}

The Abotani groups of tribes of Arunachal Pradesh have both theoretical and practical dimension knowledge and skills related to effective conservation and sustainable utilization of the plant bioresources of their traditional biocultural landscape. Some of the plant species recorded are also used for construction, handicrafts and even used to performing traditional rituals during indigenous socio-cultural occasion such as local rituals, festival and marriage ceremony. Some of the culturally significant plant species used by the 05 tribes are Calamus leptospadix, Calamus acanthospathus, Saccharum spontaneum, Saurauia punduana and Dendrocalamus hamiltonii.

\section{CONCLUSION}

It is concluded that the Abotani tribes of Arunachal Pradesh are rich in forest, agricultural and ethnobotanical resources. These tribes have both theoretical and practical dimension knowledge and skill related to effective conservation, harvesting and sustainable utilization of the significant ethnobotanical resources found in their traditional biocultural landscape which is worth learning and emulating. The diversity of ethnobotanical plant species used by the Abotani tribes of 11 districts ranges from food, medicinal and traditional ethnopharmacology to ritual, handicraft and house construction. All the 45 species reported from Abotani Biocultural Landscape $(\mathrm{ABL})$ are commonly and frequently used by all the 05 Abotani tribes which are mostly found in tropical and subtropical climatic regime while few species are found to grow in temperate climatic regime. The food and medicinal plants frequently harvested are economically and commercially profitable species which can be further encouraged and be promoted in the rural localities for food, nutritional and medicinal security. Conservation of these significant ethnobotanical species in the community forest land and jhum land should be encouraged through village level stakeholders for ensuring sustainable and stable harvesting and supply chain of food, medicinal and cultural raw materials in the market.

\section{Acknowledgements}

The authors are thankful to the Department of Biotechnology, GoI New Delhi for funding support to the Rajiv Gandhi University, Arunachal Pradesh, and National Institute of Technology -Arunachal Pradesh through DBT Twinning and DBT NER programme. The authors deeply acknowledges the Board of Research in Nuclear Science (BRNS), Department of Atomic Energy (DAE), BARC (Trombay) for funding support to RGU; SERB - Department of Science and Technology, GoI and GBPNIHESD Almora under IERP scheme 
for funding support to NIT-AP. All the authors are deeply thankful to potential local informants for their logistic help and support rendered during field visit in rural localities in Abotani Biocultural Landscape (ABL) in 11 districts of Central Arunachal Pradesh. Authors deeply acknowledge the infrastructure, administrative and logistic support rendered by the competent authority of RGU, Rono Hills, Doimukh, and NIT-Arunachal Pradesh.

\section{LITERATURE CITED}

Anonymous 2011. Census report: Arunachal Pradesh. Ministry of Home Affairs, Government of India. Pp. 5 - 20.

Bipul C.H; Yanka, H; Gaottham, G; Tag, H \& Das, A. K. 2017. Anti-Diabetic Plants Used by Apatani Tribe of Arunachal Pradesh, India. J. Biores. 4(2): 73 - 79.

Boko, N. \& Narsimhan, D. 2014. Rapid survey of plants used by Adi tribe of BosingBanggo, East Siang District, Arunachal Pradesh, India. Pleione 8(2): 271 - 282.

Chowdhery, H.J; Giri, G.S; Pal, G.D; Pramanik, A \& Das, S.K. 2009. Materials for the flora of Arunachal Pradesh. Vol. III. Botanical Survey of India, Kolkata.

Das, A.K.1986. Ethnobotany of East Siang District of Arunachal Pradesh. PhD thesis, Gauhati University, Guwahati (Unpublished).

Giri, G.S.; Pramanik, A. \& Chowdhery, H.J. 2008. Materials for the Flora of Arunachal Pradesh. Vol. II. Botanical Survey of India, Kolkata.

Gode, K.; Kanwal, K.S. \& Yama, L. 2018. Ethnomedicinal plants used by Galo Community of West Siang district, Arunachal Pradesh. Intn. J. Res. Appl. Sci. Engin. Tech. 6(1): $438-444$.

Hajra, P.K.; Verma, D.M. \& Giri, G.S. 1996. Materials for the Flora of Arunachal Pradesh. Vol. I. Botanical Survey of India, Calcutta.

Hooker, J.D. 1872 - 1897. The Flora of British India, Vols. 1 - 7. L. Reeve \& Co Ltd, Ashford, Kent. London.

Jain, S.K. \& Rao, R.R. 1977. A Hand Book of Field and Herbarium Methods, Pp $1-70$. Today \& tomorrow's Printers \& Publishers, New Delhi.

Kanjilal, U.N; Das, A.; Kanjilal, P.C.; Purkaystha, C.; De, R.N. \& Bor, N.L. $1934-1940$. Flora of Assam (Vols. I - V). Govt of Assam Press, Shillong.

Kaul, K.N. \& Haridasan, K. 1987. Forest types of Arunachal Pradesh: A preliminary study. J. Econ. Taxo. Bot. 9(2): 379.

Mao, A.A \& Hynniewta, T.M. 2000: Floristic diversity of North East India. J Assam Sci. Soc. 41(4): $255-266$.

Martin, G.J. 2008. Ethnobotany: A Methods Manual, People and Plants Conservation Series. Earthscan, UK and USA. Pp. $10-160$.

Mittermeier, R.A.; Gil, P.R.; Hoffmann, M.; Pilgrim, J.; Brooks, T.; Mittermeier, C.G.; Lamoreux, J.; Da Fonseca, G.A.B. 2005. Hotspots Revisited: Earth's Biologically Richest and Most Endangered Terrestrial Ecoregions. Conservation International.

Momang, T; Das, A.P \& Tag, H. 2018a. A new species of Panax L. (Araliaceae) from Arunachal Pradesh, India. Pleione 12(2): 315 - 321.

Momang, T; Dipankar, B; Rinyo, R; Tag, H. 2018b. Wild food plant resources of Komkar Adi tribe of Upper Siang district in Arunachal Pradesh, India. Bull.Arunachal For. Res. 33(2): 27 - 35 . 
Murtem, G. 2000. Common vegetable of Nyishi tribe of Arunachal Pradesh. Arunachal For. News 18: $64-66$.

Murtem, G. \& Chaudhry, P. 2016. An ethnobotanical study of medicinal plants used by the tribes of Upper Subansiri District of Arunachal Pradesh, India. Amer. J. Ethnomed. 3(3): $35-49$.

Myers, N; Russell, A.M; Cristina, G.M; Gustavo AB Da Fonseca \& Jennifer, K. 2000. Biodiversity hotspots for conservation priorities. Nature 403(6772): $853-858$.

Nabam N.H. 2013. The customary laws of the Thanyi Groups of Tribes of Arunachal Pradesh. Author Press, New Delhi. Pp. 9 - 127.

Omem, R; Rajiv, M \& Tag, H. 2016. Ethnobotany of the Galo community of Arunachal Pradesh, India. Pleione 10(2): 248 - 261.

Pal, G.D. 1993. Flora of Lower Subansiri District, Arunachal Pradesh, India. Ph.D thesis, University of Calcutta, Kolkata. (Unpublished).

Pallabi, K.; Tag, H; Sarma, H.N. \& Das, A.K. 2014. Evaluation of Nutritional Potential of Five Unexplored Wild Edible Food Plants from Eastern Himalayan Biodiversity Hotspot Region (India). Intn. J. Biol. Life Sci. Engin. 8(3): 1 - 4.

Rinyo, R; Momang, T; Pallabi, K.H. \& Tag, H. 2018. Ethnobotanical resources and traditional skills prevalent among the Tagin community of Arunachal Pradesh, India. Pleione 12(2): $265-274$.

Showren, T. 2009. The Nyishi of Arunachal Pradesh: An ethnohistorical study. Regency Publications (Division of Daya Publishing House), New Delhi. Pp. 3 - 98.

Tag, H. \& Das, A.K. 2007. Significant plant used by the Nyishi tribe of Arunachal Pradesh, Northeast India. In: A.P. Das \& A.K. Pandey (eds.), Advances in Ethnobotany. Bishen Singh and Mahindra Pal Singh. Dehradun. Pp. 43 - 50.

Tag, H; Das, A.K. \& Pallabi, K. 2005. Plants used by Hill Miri tribe of Arunachal Pradesh in ethnofisheries. Indian J. Trad. Knowl. 4(1): 57 - 64.

Tag, H; Murtem, G; Das, A.K. \& Singh, R.K. 2008. Diversity distribution of ethnobotanical plants used by Adi tribe of East Siang district of Arunachal Pradesh, India. Pleione 2(1): $123-136$.

Tag, H. 2007. A systematic study of plants of ethnomedicinal importance used by the Khamti Tribe of Arunachal Pradesh. Ph.D. thesis, Rajiv Gandhi University, (unpublished).

Tag, H. 2014. Biodiversity, Traditional Knowledge, Intellectual Property Rights and Benefit Sharing in the Context of Northeast India. In: M.C. Behera \& B. Jumyir (eds.), Resources, Tribes and Development. Rawat Publications, Jaipur. Pp. 259 - 279.

Tag, H. 2017. Heritage of the Kamle District, Arunachal Pradesh. A concept guide for district vision document 2030. Published by Department of Botany, Rajiv Gandhi University, Rono Hills, Doimukh, Arunachal Pradesh. Pp. 4 - 16.

http://www.theplantlist.org

http://www.plantsoftheworldonline.org/ 